\title{
Dotación fonoaudiológica para la atención de pacientes adultos en hospitales de alta complejidad en Chile
}

\author{
Felipe Rosales ${ }^{\mathbf{a}, *}$, Axel Pavez ${ }^{\mathbf{b}}$, Ariela González ${ }^{\mathbf{c}}$, Ignacio González ${ }^{\mathbf{a}}$ \\ ${ }^{a}$ Hospital San Juan de Dios, Chile \\ bospital de Urgencia Asistencia Pública, Chile \\ ' Hospital San José, Chile
}

\section{RESUMEN}

A nivel internacional se propone un mínimo de un profesional fonoaudiólogo por cada diez camas críticas o más, dependiendo de la complejidad de cada institución. En Chile no existen lineamientos similares, no obstante, el Ministerio de Salud ha generado recomendaciones para el cálculo de la brecha profesional según número y complejidad de las camas hospitalarias. El objetivo de este estudio es estimar el número de fonoaudiólogos y horas de contratación teóricas requeridas para la atención de pacientes adultos hospitalizados en instituciones públicas de alta complejidad en Chile. Para ello se realizó un estudio descriptivo, observacional, transversal. Se incluyeron todos los hospitales públicos de alta complejidad del país, excluyendo aquellos pediátricos y psiquiátricos. Como instrumentos se utilizaron el documento "Modelo de Gestión Red de Rehabilitación" del Ministerio de Salud, la base de datos actualizada del total de camas de la red hospitalaria y el software Microsoft Excel. El estudio fue aprobado por Comité Ético Científico del Hospital San Juan de Dios. En total 59 de 188 hospitales existentes en Chile fueron incluidos. De acuerdo con el análisis realizado basado en el número de camas en Chile, se requieren aproximadamente 269 fonoaudiólogos contratados 44 horas semanales para la atención de pacientes adultos en hospitales de alta complejidad. De ellos 104 se ubican en la Región Metropolitana; alrededor de 31 en la Región del BíoBío y cerca de 24 en la Región de Valparaíso. Se sugiere la revisión de las orientaciones ministeriales y la realización de un segundo estudio sobre la brecha profesional existente en Chile con los datos obtenidos.

\section{Speech and language therapists for attending adult patients in high-complexity hospitals in Chile}

\section{ABSTRACT}

International institutions have suggested one Speech and Language Therapist per ten critical beds, and possibly even more for high-complexity institutions. However, there are no similar recommendations in Chile, although the Ministry of Health has developed recommendations to calculate the number of professionals required according to the number and type of clinical bed. The aim of this study is to describe the number of Speech and Language Therapists along with the number of theorical working hours per week required for the attention of hospitalised adult patients in high-complexity hospitals in Chile, for which a descriptive, observational and transversal study was performed. High-complexity hospitals were included, excluding psychiatric and paediatric institutions. Microsoft Excel, the "Modelo de Gestión Red de Rehabilitación" guideline from the Ministry of Health, and the updated database of the clinical network beds were also used. This study was previously approved by a Research Ethics Committee from Hospital San Juan de Dios, and a total of 59 out of 188 hospitals were included. According to the analysis based on features of the clinical beds, 269 Speech and Language Therapists are required for 44 hours per week to attend adult patients in high-complexity hospitals across the country. Of the 269 professionals, 104 are needed in the Metropolitan region, approximately 31 in the Bío-Bío region and circa 24 in the Valparaíso region. According to these results, it is suggested the Ministry review its guidelines and undertake a further project in order to identify the current professional gap in Chile.

*Autor/a correspondiente: Felipe Rosales

Email:frosalestillo@gmail.com 


\section{INTRODUCCIÓN}

En la actualidad, el sistema de salud chileno está concebido como una red integrada de establecimientos de salud, cada uno con distinto tamaño, perfil, organización interna y misión particular (Ministerio de Salud, 2007). El fonoaudiólogo se ha integrado en los distintos niveles de atención del sistema de salud, con mayor preponderancia en el nivel secundario y terciario, aportando desde sus conocimientos y adaptándolos al contexto en donde se encuentre (Vega et al., 2017).

En el caso de la atención en nivel terciario de salud, es decir, hospitales de alta complejidad, el fonoaudiólogo se integra a los equipos de rehabilitación multidisciplinarios, los cuales son clave al considerar la eficiencia del cuidado de los usuarios, de los procesos diagnósticos y de las terapias (Needham, 2008). Ello, en distintos contextos y servicios de hospitalización incluyendo servicios de urgencia, unidades de pacientes críticos, geriátricos, entre otros (Cambridge University Hospital, 2019; Da Costa \& Guimarães, 2012; McRae et al., 2019; Royal College of Speech \& Language Therapist, 2019).

La evidencia muestra que la rehabilitación multidisciplinaria precoz e intensiva mejora las condiciones de alta y acorta las estadías hospitalarias. En concreto, cuando esta es aplicada en las primeras etapas disminuye los días de hospitalización $\mathrm{y}$ contribuye a reducir el riesgo de discapacidad y/o el grado de dependencia final (Ministerio de Salud, 2019). En este contexto, el fonoaudiólogo contribuye en las primeras etapas principalmente a través del manejo e intervención de los trastornos de la deglución y comunicación. Ello tiene un impacto a nivel de salud pública, ya que las alteraciones comunicativas y deglutorias generan consecuencias negativas tanto a corto como a largo plazo. Específicamente, la disfagia orofaríngea aumenta el riesgo de neumonía aspirativa, malnutrición y deshidratación (Allen et al., 2019), así como el riesgo de reingreso hospitalario y mortalidad (Altman et al., 2010; Attrill et al., 2018). La repercusión económica de la disfagia orofaríngea es considerada elevada para los sistemas de salud de diversos países debido a efectos colaterales como aumento en la estadía hospitalaria, necesidad de intervención por un equipo de salud más amplio, mayor uso de insumos, entre otros (Duncan et al., 2019). Por su parte, los costos directos asociados a los trastornos en la comunicación (lenguaje, voz, audición y habla) no han sido tan ampliamente estudiados (Flowers et al., 2016).

Considerando la relevancia de la intervención fonoaudiológica hospitalaria y la necesidad de reducir brechas en relación a recursos humanos, se han generado recomendaciones internacionales sobre el número de fonoaudiólogos requeridos a nivel hospitalario. El Royal College of Speech \& Language Therapist (RCSLT) (2019) Royal College of Speech \& Language Therapist (RCSLT) y la Intensive Care Society (ICS) (2019) recomiendan un mínimo de un fonoaudiólogo por cada diez camas críticas; recomendación que para el ICS podría ser mayor dependiendo de la complejidad y servicios entregados por cada institución. Por su parte, el National Health Service (2019) de Inglaterra señala que se espera que exista un fonoaudiólogo contratado cinco días a la semana para la atención de pacientes críticos, siendo deseable cobertura fonoaudiológica los siete días de la semana.

En Chile no existen datos publicados sobre el número de fonoaudiólogos que se desempeñan en atención hospitalaria, tampoco se cuenta con una guía nacional sobre lineamientos de fonoaudiología en el área, siendo lo más cercano el documento "Modelo de Gestión Red de Rehabilitación" (Ministerio de Salud, 2019), que corresponde a una propuesta emanada desde la Unidad de Rehabilitación de la División de Gestión de Redes Asistenciales (DIGERA) del Ministerio de Salud (MINSAL). La propuesta Ministerial tiene como objetivo principal direccionar el desarrollo de la rehabilitación en los distintos niveles de atención del sistema público, considerando para ello aspectos técnicos, de recursos humanos, equipamiento, espacios y roles de cada disciplina. Para la estimación de recurso humano profesional se utilizaron estándares extraídos de estudios internacionales (Cartmill et al., 2012; Ward et al., 2012) relacionados a los recursos profesionales necesarios para enfrentar los desafíos de la rehabilitación en hospitales (por número y tipo de cama referida a especialidades). Dichos parámetros fueron ajustados a la clasificación institucional del MINSAL que considera el uso progresivo e indiferenciado en especialidades. La estimación de recurso humano profesional no médico incluye la atención de todos los profesionales de rehabilitación del hospital en atención abierta y cerrada. Para su distribución de horas se propone que el $70 \%$ esté destinada para la atención cerrada mientras que el $30 \%$ para la atención abierta.

En base a lo expuesto, nace como pregunta de investigación ¿Cuántos son los fonoaudiólogos necesarios para la atención de pacientes adultos en Hospitales de alta complejidad en Chile? El presente trabajo tiene por objetivo estimar el número y horas de contratación teóricas de fonoaudiólogos requeridos para la atención de pacientes adultos hospitalizados en instituciones públicas de alta complejidad, en base al número de camas reportado en hospitales de alta complejidad a nivel nacional (2019). 


\section{MATERIALES Y MÉTODOS}

\section{Diseño}

Estudio descriptivo, observacional, de corte transversal.

\section{Población, muestra y unidad de análisis}

El universo corresponde a los hospitales públicos de la red asistencial, mientras que la muestra está conformada por hospitales públicos de alta complejidad que realizan atención a población adulta. Por su parte, la unidad de análisis corresponde a las camas hospitalarias.

\section{Criterios de inclusión y exclusión}

Como criterio de inclusión se consideraron todos los hospitales públicos de alta complejidad pertenecientes a los 29 servicios de salud del país. Se excluyeron las instituciones que realizan atención exclusiva de población pediátrica o psiquiátrica, así como camas de atención obstétrica, pediátrica, neonatológica, psiquiátrica, socio-sanitarias $\mathrm{y}$ de pensionado; por no ser atingentes a los objetivos del estudio.

\section{Instrumentos}

Software Microsoft Excel ${ }^{\circledR} 2016$ para la creación de la base de datos y documento "Modelo de Gestión Red de Rehabilitación" (Ministerio de Salud, 2019) para el cálculo de número de profesionales.

\section{Procedimientos}

En primer lugar, se solicitó a DIGERA del MINSAL información actualizada a diciembre de 2019 del número total de camas y complejidad de las mismas, disponibles en la red terciaria de salud pública. En segundo lugar, se creó una base de datos en Microsoft Excel ${ }^{\circledR}$ especificando los ítems: Servicio de Salud (SS), región, nombre del Hospital, número total de camas del Hospital, número de camas en Unidad de Paciente Crítico adulto (CUPCa), número de camas medias adultos (CMa) y número de camas básicas adultos $(\mathrm{CBa})$. En tercer lugar, siguiendo la fórmula declarada en el documento "Modelo de Gestión Red de Rehabilitación" (Ministerio de Salud, 2019), se traspasó la fórmula de la tabla de cálculo de recurso humano profesional según tipo de cama hospitalaria de dicho documento considerando el número necesario de fonoaudiólogos requeridos por institución. Como fue mencionado, no se consideraron camas de atención obstétrica, pediátrica, neonatológica, psiquiátricas, socio-sanitarias ni camas de pensionado, por no ser atingentes a los objetivos del estudio. El número total de $\mathrm{CBa}$ corresponde a la sumatoria de camas adultas del área médica y médica-quirúrgica básicas; el número total de CMa corresponde a la sumatoria de camas adultas del área médica y médica-quirúrgica medias; mientras que el número de camas de UPCa corresponde a la sumatoria corresponde a la sumatoria de camas adultas de cuidados intensivos (UCI) y tratamiento intermedio (UTI). Finalmente, con todos los datos anteriores se calculó el número necesario de fonoaudiólogos requeridos por institución, en base a la fórmula declarada en el documento Ministerial como se detalla en la Tabla 1.

Tabla 1. Propuesta cálculo de RRHH Fonoaudiológico según fórmula del "Modelo de Gestión Red de Rehabilitación" del (Ministerio de Salud, 2019).

\begin{tabular}{lc}
\hline $\begin{array}{l}\text { Tipo complejidad de } \\
\text { cama }\end{array}$ & $\begin{array}{c}\text { Fórmula para estimación de RRHH } \\
\text { Fonoaudiológico }\end{array}$ \\
\hline Básica $(\mathrm{CB})^{*}$ & $\left(\mathrm{~N}^{\circ} \mathrm{CB} / 10\right) * 0,06$ \\
Media $(\mathrm{CM})$ & $\left(\mathrm{N}^{\circ} \mathrm{CM} / 10\right) * 0,23$ \\
$\mathrm{UPC}$ adulto $(\mathrm{CUPCa})$ & $\left(\mathrm{N}^{\circ} \mathrm{CUPCa} / 6\right) * 0,46$ \\
\hline
\end{tabular}

(*) De acuerdo a lo declarado por el MINSAL para el cálculo estos factores se aplican sólo al 30\% del total de camas básicas (CB), al 70\% se le aplican los factores de cama media ya que, por dependencia y necesidades de apoyo, tienen requerimientos mayores de rehabilitación.

\section{Análisis de datos}

Al tratarse de un cálculo simple mediante la fórmula entregada por la Tabla 1, no fue necesario llevar a cabo un análisis de datos estadístico para la obtención de resultados ni realizar análisis de significancia. Por lo anterior, se utilizó exclusivamente estadística descriptiva, traspasando la tabla y la fórmula a una hoja de cálculo del software Microsoft Excel ${ }^{\circledR}$ para obtener los resultados. Considerando que de acuerdo a las directrices estipuladas en el estatuto administrativo (documento legal que rige a los funcionarios públicos), se considera personal diurno como aquellos profesionales contratados por una jornada laboral de 44 horas semanales, el número de horas fonoaudiológicas totales se obtuvo multiplicando la cantidad de profesionales fonoaudiólogos estimados por 44 horas, que corresponden a una jornada profesional completa, siendo las fracciones de 33 horas, 22 horas y 11 horas las únicas posibles y que constituyen jornadas parciales. Para realizar la propuesta de jornada, se realizó el análisis de datos y la conversión delas horas totales a su jornada correspondiente, considerando para ello: un valor de 0,25 equivalente a 11 horas profesionales semanales, 0,5 equivalente a 
22 horas semanales, 0,75 equivalente a 33 horas semanales y 1 el equivalente a una jornada profesional completa de 44 horas semanales. Para aquellos casos que se encontraron entre las unidades fraccionadas, los valores se aproximaron al límite inferior, vale decir, si el total equivalente fue 3,65 fonoaudiólogos, se interpretó como 3 profesionales de 44 horas y los decimales como 1 de 22 horas. Es importante destacar que para efectos del cálculo se presentarán brechas entre el número de horas totales y el número de horas propuestas por jornada (variable continua), entiendo que este último punto considera número de profesionales (variable discreta) que debe cumplir con dicha cantidad de horas.

\section{Consideraciones éticas}

Este estudio considera información de carácter público registrada en base de datos de la División de Gestión de Redes Asistenciales del MINSAL y cuenta con aprobación del Comité Ético Científico del Hospital San Juan de Dios de Santiago, con fecha 30 de abril de 2020 .

\section{RESULTADOS}

En la base de datos aportada por DIGERA, con fecha de última actualización a diciembre de 2019, se encontraron 188 hospitales públicos pertenecientes a los 29 Servicios de Salud del país. De ellos 64 correspondían a hospitales de alta complejidad por lo que fueron incluidos en la muestra. Según criterios de inclusión y exclusión, la muestra final a analizar quedó conformada por 59 instituciones. La Tabla 2 (ver anexo) resume en detalle el número y tipo de camas por cada institución en relación al Servicio de Salud y región correspondiente. Junto a ello, el número total de fonoaudiólogos requeridos por hospital según número de horas calculadas y la correspondiente propuesta de jornada laboral para cubrir dichas horas necesarias de fonoaudiología.

\section{Número de fonoaudiólogos}

Del cálculo realizado y de acuerdo a lo propuesto por el Ministerio de Salud (2019), existe un requerimiento teórico a nivel nacional aproximado de 269 fonoaudiólogos $(n=268,86)$ para dar cobertura a las demandas de los usuarios adultos de los hospitales de alta complejidad según número de camas disponibles.

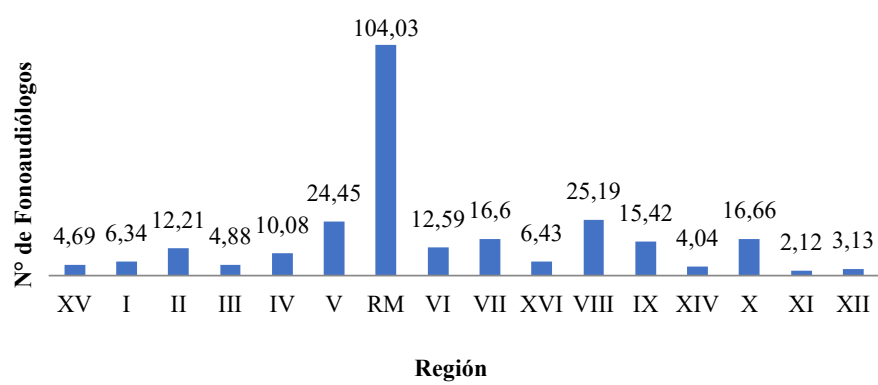

Figura 1. Número total de requerimiento estimado de fonoaudiólogos a nivel nacional, distribuidos por región.

Del total nacional estimado, de acuerdo a la Figura 1, la mayor demanda de fonoaudiólogos se concentran en los diferentes hospitales de los SS de la Región Metropolitana (RM) representando un 38,69\% del total nacional $(n=104,03)$; le siguen los hospitales de los SS de la Región del BíoBío (VIII) con un $9,37 \%(n=25,19)$ y en tercer lugar los hospitales de los SS de la Región de Valparaíso (V) con un 9,09\% $(n=24,45)$. El mínimo valor lo obtiene el SS de la Región de Aysén (XI) con un 0,79\% $(n=2,12)$. El resto de los hospitales de los SS de las diferentes regiones fluctúan con valores de entre $1,16 \%$ (XII región) y $6,20 \%$ (X región).

De los 59 hospitales analizados, los 10 con mayor necesidad de fonoaudiólogos a nivel nacional son (Tabla 2, ver Anexo): el Hospital Dr. Hernán Henríquez Aravena de Temuco con un número aproximado de 13 profesionales $(n=12,59)$; cercano a 11 fonoaudiólogos el Hospital Barros Luco Trudeau $(n=10,96)$ junto al Hospital de Talca $(\mathrm{n}=10,74)$; y alrededor de 10 profesionales el Hospital Regional de Rancagua $(n=10,39)$, el Hospital de Puerto Montt $(n=10,32)$, el Hospital San Juan de Dios de Santiago $(\mathrm{n}=10,12)$, el Hospital Clínico Regional Dr. Guillermo Grant Benavente $(n=10,04)$, el Complejo hospitalario Dr. Sótero del Río $(n=9,76)$, el Hospital de Urgencia Asistencia Pública $(n=9,61)$ y Hospital Dr. Leonardo Guzmán (n=9,57).

\section{Horas totales de fonoaudiólogos}

Del cálculo realizado (Tabla 2, ver Anexo), se requieren aproximadamente 11.830 horas $(\mathrm{h})$ totales semanales de atención fonoaudiológica a nivel país, para lograr dar cobertura a los usuarios adultos que se encuentran en los hospitales de alta complejidad de la red pública asistencial. Del total nacional estimado, de acuerdo a la Figura 2, la mayor cantidad de horas necesarias se concentran en los diferentes hospitales de los SS de la Región Metropolitana (RM) representando un 38,69\% del total nacional $(\mathrm{n}=4577,32 \mathrm{~h})$; le siguen hospitales de los $\mathrm{SS}$ de la 
Región del BíoBío (VIII) con un 9,37\% ( $\mathrm{n}=1108,36 \mathrm{~h}$ ) y en tercer lugar los hospitales de los SS de la Región de Valparaíso (V) con un $9,09 \%$ ( $\mathrm{n}=1075,8 \mathrm{~h})$. El mínimo valor lo obtiene el SS de la Región de Aysén (XI) con un 0,79\% (n=93,28 h). El resto de los hospitales de los SS de las diferentes regiones fluctúan con valores de entre $1,16 \%$ (XII región) y $6,20 \%$ (X región). La cantidad de horas totales semanales fonoaudiológicas requeridas por cada institución, se pueden observar en la Tabla 2 (ver Anexo). Considerando que la cantidad de horas es un valor directamente proporcional al número de fonoaudiólogos requeridos por institución, los 10 hospitales con mayor cantidad de horas semanales coinciden con lo expuesto en el punto anterior. A continuación, se detallan éstos mostrando en paréntesis el total de horas semanales: en primer lugar el Hospital Dr. Hernán Henríquez Aravena de Temuco ( $\mathrm{n}=554 \mathrm{~h}$ ); luego el Hospital Barros Luco Trudeau ( $n=482$ h), el Hospital de Talca ( $n=473$ h); el Hospital Regional de Rancagua $(\mathrm{n}=457 \mathrm{~h})$; el Hospital de Puerto Montt ( $\mathrm{n}=454 \mathrm{~h}$ ); el Hospital San Juan de Dios de Santiago $(\mathrm{n}=445 \mathrm{~h})$; el Hospital Clínico Regional Dr. Guillermo Grant Benavente $(n=442 \mathrm{~h})$; el Complejo Hospitalario Dr. Sótero del Río ( $\mathrm{n}=429$ h); el Hospital de Urgencia Asistencia Pública ( $\mathrm{n}=423$ h) y el Hospital Dr. Leonardo Guzmán ( $n=421$ h).

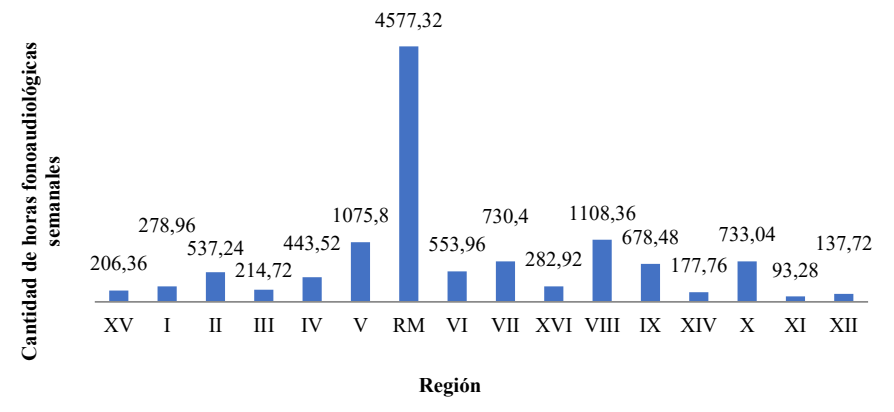

Figura 2. Cantidad de horas fonoaudiológicas totales estimadas a nivel nacional, distribuidos por región.

\section{Propuesta de distribución por jornada}

Para dar respuesta a las demandas en relación al número de profesionales y horas necesarias, se plantea en la Figura 3 una distribución de fonoaudiólogos en base a jornadas de 11, 22, 33 y 44 horas semanales, según región y total país. El 84,15\% de los fonoaudiólogos a nivel país ( $\mathrm{n}=239)$ debería desempeñarse en jornada de 44 horas; mientras que un 7,04\% $(n=20)$ lo debería realizar en jornada de 22 horas, $4,58 \%(n=13)$ en jornada de 33 horas y $4,23 \%(n=12)$ en jornada de 11 horas.

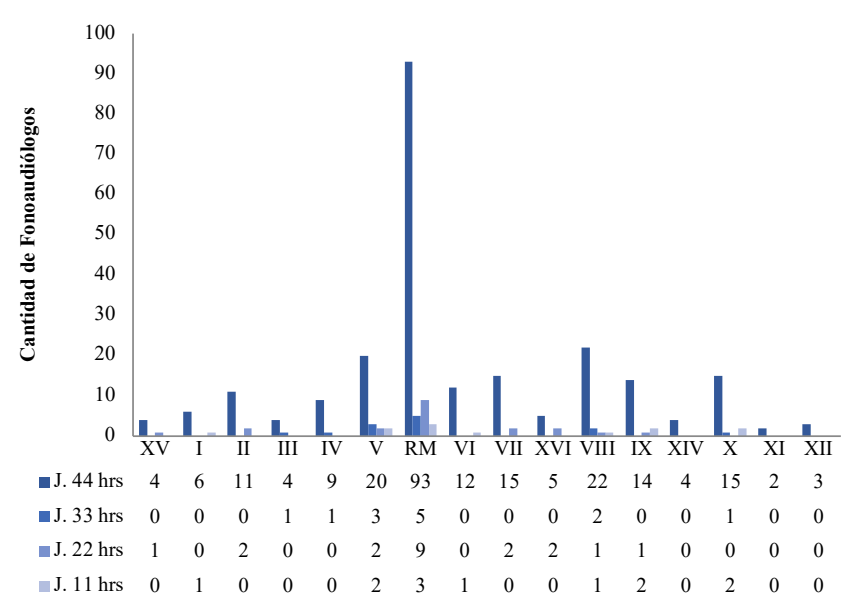

Figura 3. Propuesta de distribución de fonoaudiólogos por jornada de 11, 22, 33 y 44 horas según región y total país.

\section{DISCUSIÓN}

Este estudio tuvo por objetivo estimar el número y horas de contratación teóricas de fonoaudiólogos requeridos para la atención de pacientes adultos hospitalizados en instituciones públicas de alta complejidad. Se cuantificó un estimado de número de horas teóricas semanales y distribución por jornada. Considerando la característica descriptiva de la investigación y sus resultados, existen puntos relevantes a destacar.

Uno de los primeros aspectos se encuentra relacionado a la necesidad de fonoaudiólogos en cada hospital de alta complejidad y sus servicios de salud correspondiente. Al realizar la suma de todos los Servicios de Salud, es la región Metropolitana la que concentra la mayor necesidad de fonoaudiólogos a nivel país. Sin embargo, al realizar el desglose individual por institución, son los hospitales regionales de Temuco y Talca, respectivamente, quienes presentan mayor requerimiento específico. Esto se debe a que la región Metropolitana concentra un mayor número de recintos hospitalarios de alta complejidad en comparación a otras regiones, lo cual la posiciona en primer lugar en cuanto a necesidad de fonoaudiólogos. Sin embargo, el desglose específico evidencia la relevancia de hospitales pertenecientes a otras regiones del país, las cuales no corresponden necesariamente a aquellas con mayor población después de la región Metropolitana según cifras del Instituto Nacional de Estadísticas (2017). A diferencia de lo que ocurre en la RM donde hay varios hospitales de alta complejidad, en otras regiones del país este número es menor siendo necesario para las instituciones de alta complejidad 
funcionar como centro de referencia regional y así, absorber las necesidades de la región completa.

En esta misma línea, también se puede observar que las tres primeras regiones que requieren mayor número de profesionales coinciden con las que a nivel nacional presentan un mayor número de habitantes. No obstante, a pesar que la región de Valparaíso concentra mayor población en comparación a la región del Bíobío, esta última presenta un mayor requerimiento de fonoaudiólogos. Un fenómeno similar ocurre con las regiones que presentan menor número de habitantes. Todo lo anterior, se explica por la vía de cálculo profesional propuesta por el Ministerio de Salud (2019) que se basa exclusivamente en la cantidad de camas hospitalarias y su complejidad, no considerando datos propios de estadística poblacional nacional. Por lo anterior, la fórmula permite establecer el número de fonoaudiólogos teórico e ideal para cada establecimiento de salud, pero en ningún caso debe entenderse como dotación óptima para la población total a la que dicha institución entrega cobertura. Además, es necesaria la discusión en relación a este cálculo, considerando que Chile se encuentra bajo el promedio de camas hospitalarias disponibles por habitantes según la Organización para la Cooperación y el Desarrollo Económico (2014), presentando un promedio de 2,1 camas por cada 1.000 habitantes versus el promedio de la organización que corresponde a 4,7. Por ello, el cálculo resultante podría no ser un estimativo de la necesidad real.

La estimación de jornadas laborales requeridas para cubrir las horas fonoaudiológicas semanales necesarias da cuenta que la mayoría de los profesionales deberían ser contratados en jornadas completas semanales (44 horas), seguido muy de lejos por aquellos contratados por media jornada ( 22 horas). A pesar que esta estimación fue realizada siguiendo criterios matemáticos, es importante destacar que se deberían incluir otro tipo de variables para generar esta distribución, especialmente aquellas relacionadas con actividades no clínicas, por ejemplo: la contemplación de horas legales de colación para aquellas personas contratadas por media jornada, así como la cantidad de horas administrativas que varía entre un profesional de 44 horas versus otro con jornada laboral menor.

En cuanto a la distribución de horas fonoaudiológicas entre atención abierta y cerrada, el documento ministerial (Ministerio de Salud, 2019) plantea como ideal que en instituciones de alta complejidad el $70 \%$ de las prestaciones fonoaudiológicas sea en atención cerrada con una intensidad de hasta dos atenciones diarias, mientras que el $30 \%$ en atención abierta. Lo anterior no se encuentra descrito en los estudios referenciados por el Ministerio para desarrollar la tabla para el cálculo profesional (Cartmill et al.,
2012; Ward et al., 2012), así como tampoco se cuenta actualmente con un modelo que permita estimar la frecuencia de las atenciones fonoaudiológicas en pacientes hospitalizados y así poder estimar de manera más fidedigna las horas reales requeridas. Adicionalmente, la cifra obtenida tras la aplicación de la fórmula no sigue la línea de las recomendaciones internacionales descritas en relación a la dotación de fonoaudiólogos en el ámbito hospitalario, siendo menor a lo propuesto por el RCSLT (2019) y la ICS (2019). En la misma línea, Ward et al. (2010) indica que por cada 25 pacientes hospitalizados se requiere 1,5 fonoaudiólogos a tiempo completo, esto es, un cargo de 44 horas y uno de 22 horas independiente del nivel de complejidad de la atención. Asignar el 30\% de las horas a atención abierta aumenta aún más la diferencia con dichas recomendaciones.

El presente estudio constituye un aporte, tanto para el desarrollo de la profesión a nivel nacional como hacia los equipos de atención clínica de las instituciones públicas de alta complejidad, entregando información y orientación teórica sobre la dotación de fonoaudiólogos necesarios según el nivel de complejidad de camas de los hospitales para la determinación de su brecha profesional, información que hasta la fecha no se encuentra disponible ni publicada en el medio nacional, tanto en revistas científicas como en lineamientos del propio MINSAL.

Una de las principales limitaciones del estudio lo constituye la actualización de la base de datos de DIGERA correspondiente a diciembre de 2019, por lo cual podría variar el cálculo según apertura de nuevas camas o instituciones durante el transcurso de la investigación. En este sentido, es importante considerar las cifras expuestas sólo como una orientación pertinente a la realidad correspondiente a esa fecha. Por otro lado, este estudio fue concebido previo al inicio de la pandemia COVID-19, la que ha implicado modificaciones en número y complejidad de camas de los recintos hospitalarios del país. Hasta el momento de redacción de este artículo, la permanencia de estos cambios todavía no se ha confirmado, sin embargo, es probable que en algunos casos sí lo sean por lo que el número de horas fonoaudiológicas calculada deberá ser revisada y modificada.

Es importante considerar que, de acuerdo al objetivo de la investigación, no se incluyó el cálculo de fonoaudiólogos necesarios para la población infantil ni para los de hospitales de mediana y baja complejidad. Sería interesante en investigaciones futuras conocer dicho número de profesionales, como también continuar esta línea de investigación para poder contrastar las cifras aquí obtenidas con el número real de fonoaudiólogos existentes en los Hospitales públicos de alta complejidad, de este modo, poder determinar la existencia de brecha entre los 
profesionales reales contratados en las instituciones versus la dotación establecida por el MINSAL según número de camas. Junto a esto, describir otros aspectos relacionados, como la distribución de sus jornadas entre atención cerrada y abierta o la calidad contractual.

\section{AGRADECIMIENTOS}

A la Sra. Lenka Parada G. de la Unidad de Rehabilitación perteneciente a la División de Gestión de Redes Asistenciales, Subsecretaría de Redes Asistenciales del Ministerio de Salud, por su apoyo en este proyecto de investigación.

\section{REFERENCIAS}

Allen, J., Greene, M., Sabido, I., Stretton, M., \& Miles, A. (2019). Economic costs of dysphagia among hospitalized patients. The Laryngoscope, 130(4), 974-979. https://doi.org/10.1002/lary.28194

Altman, K. W., Yu, G.-P., \& Schaefer, S. D. (2010). Consequence of Dysphagia in the Hospitalized Patient: Impact on Prognosis and Hospital Resources. Archives of Otolaryngology-Head \& Neck Surgery, 136(8), 784-789. https://doi.org/10.1001/archoto.2010.129

Attrill, S., White, S., Murray, J., Hammond, S., \& Doeltgen, S. (2018). Impact of oropharyngeal dysphagia on healthcare cost and length of stay in hospital: A systematic review. BMC Health Services Research, 18(1), 594. https://doi.org/10.1186/s12913-018-3376-3

Cambridge University Hospital. (2019). Speech and Language Teams. https://www.cuh.nhs.uk/addenbrookes-hospital/services/speech-and-languageteams

Cartmill, L., Comans, T. A., Clark, M. J., Ash, S., \& Sheppard, L. (2012). Using staffing ratios for workforce planning: Evidence on nine allied health professions. Human Resources for Health, 10(1), 2. https://doi.org/10.1186/1478-4491-10-2

Da Costa, K., \& Guimarães, V. (2012). Fonoaudiologia nos serviços de urgência e emergência do Brasil: Série histórica de 2005 a 2011. Distúrbios Da Comunicação, 24(1), 69-75. https://revistas.pucsp.br/index.php/dic/article/view/9707

Duncan, S., Gaughey, J. M., Fallis, R., McAuley, D. F., Walshe, M., \& Blackwood, B. (2019). Interventions for oropharyngeal dysphagia in acute and critical care: A protocol for a systematic review and meta-analysis. Systematic Reviews, 8(1), 283. https://doi.org/10.1186/s13643-019-1196-0
Flowers, H. L., Skoretz, S. A., Silver, F. L., Rochon, E., Fang, J., Flamand-Roze, C., \& Martino, R. (2016). Poststroke Aphasia Frequency, Recovery, and Outcomes: A Systematic Review and Meta-Analysis. Archives of Physical Medicine and Rehabilitation, 97(12), 2188-2201.e8. https://doi.org/10.1016/j.apmr.2016.03.006

Instituto Nacional de Estadísticas. (2017). Resultados Censo 2017. http://resultados.censo2017.cl/

Intensive Care Society, \& The Faculty of Intensive Care Medicine. (2019). Guidelines for the Provision of Intensive Care Services:The Faculty of Intensive Care Medicine. https://ficm.ac.uk/sites/default/files/gpics-v2-final2019.pdf

McRae, J., Montgomery, E., Garstang, Z., \& Cleary, E. (2019). The role of speech and language therapists in the intensive care unit. Journal of the Intensive Care Society, 1-5. https://doi.org/10.1177/1751143719875687

Ministerio de Salud. (2007). La Red Pública de Salud. Fundamentos, Conceptos y Experiencia. http://www.bibliotecaminsal.cl/wp/wp-

content/uploads/2016/03/8.pdf

Ministerio de Salud. (2019). Modelo de Gestión Red de Rehabilitación. Ministerio de Salud. https://www.araucaniasur.cl/wp-content/uploads/2019/06/MODELODE-GESTION-RED-DE-REHABILITACION.pdf

National Health Service. (2019). Schedule 2 - The Services. https://www.england.nhs.uk/wp-content/uploads/2019/05/Adult-Critical-CareService-Specification-FINAL.pdf

Needham, D. M. (2008). Mobilizing patients in the intensive care unit: Improving neuromuscular weakness and physical function. JAMA, 300(14), 1685-1690. https://doi.org/10.1001/jama.300.14.1685

Organización para la Cooperación y el Desarrollo Económico. (2014). Estadísticas de la OCDE sobre la Salud 2014. Chile en comparación. https://www.oecd.org/els/health-systems/Briefing-Note-CHILE-2014-inSpanish.pdf

Royal College of Speech \& Language Therapist. (2019). Position Statement: Speech and Language Therapists Working in Adult and Paediatric Critical Care Units. https://www.rcslt.org/-/media/docs/clinical-guidance/rcslt-positionstatement-critical-

care.pdf?la=en\&hash=42823C17957D4848818438CBCD5DC3998EF0CDF7

Vega, Y. E., Torres, A. M., \& del Campo, M. N. (2017). Análisis del Rol del Fonoaudiólogo(a) en el Sector Salud en Chile. Ciencia \& trabajo, 19(59), 76-80. https://doi.org/10.4067/S0718-24492017000200076

Ward, A., Gutenbrunner, C., Giustini, A., Delarque, A., Fialka-Moser, V., Kiekens, C., Berteanu, M., \& Christodoulou, N. (2012). A position paper on Physical \& Rehabilitation Medicine programmes in post-acute settings. Union of European Medical Specialists Section of Physical \& Rehabilitation Medicine (in conjunction with the European Society of Physical \& Rehabilitation Medicine). Journal of Rehabilitation Medicine, 44(4), 289-298 https://doi.org/10.2340/16501977-0960 


\section{ANEXOS}

Tabla 2. Número de Fonoaudiólogos requeridos para cubrir la demanda hospitalaria de usuarios adultos según cálculo de fórmula MINSAL.

\begin{tabular}{|c|c|c|c|c|c|c|c|c|c|}
\hline Región & $\begin{array}{l}\text { Servicio de } \\
\text { Salud }\end{array}$ & Hospital & $\begin{array}{l}\mathrm{N}^{\circ} \text { total } \\
\text { de camas }\end{array}$ & $\begin{array}{c}\mathbf{N}^{\circ} \\
\mathbf{U P C a}\end{array}$ & $\begin{array}{c}\mathbf{N}^{\circ} \\
\text { CMa }\end{array}$ & $\begin{array}{l}\mathrm{N}^{\circ} \\
\mathrm{CBa}\end{array}$ & $\begin{array}{c}\mathbf{N}^{\circ} \text { FNA } \\
\text { estimado* }\end{array}$ & $\begin{array}{c}\mathbf{N}^{\circ} \text { horas } \\
\text { totales } \\
\text { FNA** }\end{array}$ & $\begin{array}{c}\text { Propuesta } \\
\text { jornada } * * * \\
(\text { FNA } x \text { h) }\end{array}$ \\
\hline \multirow[t]{2}{*}{$\mathrm{XV}$} & Arica y & Hospital Regional Dr. Juan Noé & 313 & 18 & 136 & 30 & 4,69 & 206,36 & $4 \times 44$ \\
\hline & Parinacota & Crevanni & & & & & & & $1 \times 22$ \\
\hline \multirow[t]{2}{*}{ I } & Iquique y & Hospital Dr. Ernesto Torres & 440 & 32 & 146 & 88 & 6,34 & 278,96 & $6 \times 44$ \\
\hline & Tarapacá & Galdames & & & & & & & $1 \times 11$ \\
\hline \multirow[t]{4}{*}{ II } & Antofagasta & Hospital Dr. Leonardo Guzmán & 575 & 55 & 197 & 137 & 9,57 & 421,08 & $9 \times 44$ \\
\hline & & & & & & & & & $1 \times 22$ \\
\hline & & Hospital Dr. Carlos Cisternas & 202 & 12 & 65 & 38 & 2,64 & 116,16 & $2 \times 44$ \\
\hline & & & & & & & & & $1 \times 22$ \\
\hline \multirow[t]{2}{*}{ III } & Atacama & Hospital San José del Carmen & 364 & 16 & 135 & 92 & 4,88 & 214,72 & $4 \times 44$ \\
\hline & & & & & & & & & $1 \times 33$ \\
\hline \multirow[t]{4}{*}{ IV } & Coquimbo & Hospital San Juan de Dios & 300 & 34 & 84 & 58 & 4,88 & 214,72 & $4 \times 44$ \\
\hline & & & & & & & & & $1 \times 33$ \\
\hline & & Hospital San Pablo & 301 & 20 & 24 & 159 & 3,04 & 133,76 & $3 \times 44$ \\
\hline & & $\begin{array}{l}\text { Hospital Dr. Antonio Tirado } \\
\text { Lanas }\end{array}$ & 191 & 10 & 48 & 49 & 2,16 & 95,04 & $2 \times 44$ \\
\hline \multirow[t]{15}{*}{$\mathrm{V}$} & Aconcagua & Hospital de San Camilo & 225 & 12 & 0 & 110 & 1,58 & 69,52 & $1 \times 44$ \\
\hline & & & & & & & & & $1 \times 22$ \\
\hline & & Hospital San Juan de Dios & 168 & 6 & 12 & 93 & 1,29 & 56,76 & $1 \times 44$ \\
\hline & & & & & & & & & $1 \times 11$ \\
\hline & Valparaíso & Hospital Carlos Van Buren & 499 & 36 & 141 & 173 & 7,04 & 309,76 & $7 \times 44$ \\
\hline & & Hospital Dr. Eduardo Pereira & 194 & 12 & 0 & 175 & 1,97 & 86,68 & $1 \times 44$ \\
\hline & & Ramírez & & & & & & & $1 \times 33$ \\
\hline & & Hospital Claudio Vicuña & 164 & 6 & 12 & 97 & 1,32 & 58,08 & $1 \times 44$ \\
\hline & & & & & & & & & $1 \times 11$ \\
\hline & Viña del Mar & Hospital Dr. Gustavo Fricke & 443 & 37 & 96 & 147 & 5,93 & 260,92 & $5 \times 44$ \\
\hline & & & & & & & & & $1 \times 33$ \\
\hline & & Hospital San Martín & 208 & 14 & 102 & 22 & 3,55 & 156,2 & $3 \times 44$ \\
\hline & & & & & & & & & $1 \times 22$ \\
\hline & & Hospital de Quilpué & 156 & 10 & 28 & 60 & 1,77 & 77,88 & $1 \times 44$ \\
\hline & & & & & & & & & $1 \times 33$ \\
\hline \multirow[t]{12}{*}{ XIII - RM } & Metropolitano & Complejo Hospitalario San José & 561 & 42 & 93 & 256 & 6,89 & 303,16 & $6 \times 44$ \\
\hline & Norte & & & & & & & & $1 \times 33$ \\
\hline & & Instituto Nacional del Cáncer & 81 & 8 & 0 & 73 & 1,05 & 46,2 & $1 \times 44$ \\
\hline & Metropolitano & Hospital San Juan de Dios & 574 & 76 & 139 & 182 & 10,12 & 445,28 & $10 \times 44$ \\
\hline & Occidente & & & & & & & & \\
\hline & & Instituto Traumatológico Dr. & 104 & 6 & 40 & 50 & 1,68 & 73,92 & $1 \times 44$ \\
\hline & & Teodoro Gebauer & & & & & & & $1 \times 22$ \\
\hline & & Hospital Dr. Félix Bulnes Cerda & 362 & 26 & 80 & 34 & 4,04 & 177,76 & $4 \times 44$ \\
\hline & & Hospital San José & 133 & 9 & 24 & 51 & 1,55 & 68,2 & $1 \times 44$ \\
\hline & & & & & & & & & $1 \times 22$ \\
\hline & Metropolitano & Hospital Clínico San Borja & 558 & 41 & 100 & 143 & 6,3 & 277,2 & $6 \times 44$ \\
\hline & Central & Arriarán & & & & & & & $1 \times 11$ \\
\hline
\end{tabular}


Hospital Clínico Metropolitano

El Carmen Doctor Luis Valentín

Ferrada

Hospital de Urgencia Asistencia

Pública Dr. Alejandro del Río

Metropolitano Hospital Del Salvador

Oriente

Hospital Dr. Luis Tisné B.

Instituto Nacional de

Enfermedades Respiratorias y

Cirugía Torácica

Instituto de Neurocirugía Dr.

Alfonso Asenjo

Instituto Nacional Geriátrico

Presidente Eduardo Frei

Montalva.

Metropolitano Sur

VI

O’Higgins

La Florida Dra. Eloísa Díaz

Metropolitano

Sur-Oriente

Hospital El Pino

Hospital Parroquial

Complejo Hospitalario Dr.

Sótero del Río

Hospital Padre Alberto Hurtado

Hospital Clínico Metropolitano

Hospital Regional de Rancagua

Hospital San Juan de Dios

VII Maule

Hospital San Juan de Dios

Hospital Dr. César Garavagno

Burotto

Hospital Presidente Carlos

Ibáñez del Campo

XVI Ñuble

Hospital Clínico Herminda

Martín

Hospital de San Carlos

VIII$$
310
$$$$
148
$$$$
18
$$

98

23

34

\begin{tabular}{|c|c|c|c|c|c|c|}
\hline 418 & 18 & 264 & 15 & 7,54 & 331,76 & $\begin{array}{l}7 \times 44 \\
1 \times 22\end{array}$ \\
\hline
\end{tabular}

$\begin{array}{lllllll}264 & 66 & 198 & 0 & 9,61 & 422,84 & 9 \times 44\end{array}$

$1 \times 33$$$
34
$$

$98 \quad 23$$$
73
$$

73

$$
303
$$$$
158
$$$$
79
$$$$
417
$$

39

536

178

28

$$
28
$$$$
61
$$$$
25
$$$$
470
$$$$
116
$$$$
882
$$$$
112
$$$$
405
$$$$
146
$$

14
\end{abstract}

112

405

$0 \quad 34 \quad 0$

739

79

$$
107
$$

$\begin{array}{llll}30 & 141 & 15 & 5,63\end{array}$

$\begin{array}{lllll}16 & 0 & 70 & 1,65\end{array}$

$\begin{array}{lllll}796 & 60 & 146 & 300 & 9,76\end{array}$

$\begin{array}{lllll}17 & 16 & 58 & 170 & 3,58\end{array}$

$\begin{array}{lllll}94 & 75 & 132 & 4,36\end{array}$

$\begin{array}{lllll}36 & 60 & 252 & 0 & 10,39\end{array}$

18

85

17

615

72




\begin{tabular}{|c|c|c|c|c|c|c|c|c|c|}
\hline & BíoBío & Complejo Asistencial Dr. Víctor & 524 & 35 & 157 & 99 & 6,89 & 303,16 & $6 \times 44$ \\
\hline & & Ríos Ruiz & & & & & & & $1 \times 33$ \\
\hline \multirow[t]{6}{*}{ IX } & Araucanía & Hospital Dr. Mauricio & 184 & 6 & 24 & 65 & 1,4 & 61,6 & $1 \times 44$ \\
\hline & Norte & Heyermann & & & & & & & $1 \times 11$ \\
\hline & & Hospital San José de Victoria & 179 & 10 & 0 & 110 & 1,43 & 62,92 & $1 \times 44$ \\
\hline & & & & & & & & & $1 \times 11$ \\
\hline & Araucanía Sur & Hospital Dr. Hernán Henríquez & 719 & 66 & 316 & 44 & 12,59 & 553,96 & $12 \times 44$ \\
\hline & & Aravena & & & & & & & $1 \times 22$ \\
\hline XIV & Valdivia & Hospital Base de Valdivia & 502 & 22 & 33 & 266 & 4,04 & 177,76 & $4 \times 44$ \\
\hline \multirow[t]{6}{*}{$\mathrm{X}$} & Osorno & Hospital Base San José de & 377 & 24 & 55 & 138 & 3,93 & 172,92 & $3 \times 44$ \\
\hline & & Osorno & & & & & & & $1 \times 33$ \\
\hline & Reloncaví & Hospital de Puerto Montt & 530 & 66 & 209 & 75 & 10,32 & 454,08 & $10 \times 44$ \\
\hline & & & & & & & & & $1 \times 11$ \\
\hline & Chiloé & Hospital de Castro & 129 & 19 & 41 & 2 & 2,41 & 106,04 & $2 \times 44$ \\
\hline & & & & & & & & & $1 \times 11$ \\
\hline $\mathrm{XI}$ & Aysén & Hospital Regional de Coyhaique & 160 & 14 & 38 & 28 & 2,12 & 93,28 & $2 \times 44$ \\
\hline XII & Magallanes & Hospital Clínico de Magallanes & 326 & 24 & 1 & 211 & 3,13 & 137,72 & $3 \times 44$ \\
\hline \multirow[t]{4}{*}{ Total país } & & & & & & & 268,86 & $11.829,84$ & $239 \times 44$ \\
\hline & & & & & & & & & $13 \times 33$ \\
\hline & & & & & & & & & $20 \times 22$ \\
\hline & & & & & & & & & $12 \times 11$ \\
\hline
\end{tabular}

$\mathrm{N}^{\circ}$ UPCa: Número de camas Unidad de Paciente Crítico adulto; $\mathrm{N}^{\circ} \mathrm{CMa}$ : Número de Camas Medias adulto; $\mathrm{N}^{\circ}$ CBa: Número de Camas básicas adulto; FNA: Fonoaudiólogo(a). (*) Valor aproximado a 2 decimales. Este número considera Fonoaudiólogos de jornada 44 h. (**) Valor aproximado en relación a jornada de 44 h. $(* * *)$ FNA $\mathrm{x}$ h: número de fonoaudiólogos(as) contratados por número de horas semanales. 